\title{
L'Importance des lieux et de l'espace dans Bajazet
}

\section{Alain-Philippe DURAND}

$\mathrm{E}$

n écrivant Bajazet Racine a définitivement amorcé un des tournants les plus risqués de sa carrière. Revenons en arrière et plus précisément à l'année 1672. C'est en janvier que Bajazet est représentée pour la première fois à l'Hôtel de Bourgogne ${ }^{1}$ et Racine a de quoi s'inquiéter. En effet, bousculant les règles de la "sacro sainte" Antiquité, il a non seulement basé son oeuvre sur une histoire qu'il affirme "véritable" et vieille de trente ans, ${ }^{2}$ mais il a également situé sa tragédie en Turquie. Le dramaturge a donc choisi un lieu bien moderne pour son époque. De plus, le concept du lieu et de l'espace joue un rôle central dans Bajazet. Jusqu'à cette oeuvre, que ce soit dans Britannicus, Bérénice ou Andromaque, Racine utilisait presque toujours le même schéma en ce qui concerne le lieu: un palais ou un temple cadre de la discussion et de l'action, et un port en guise d'échappatoire. Autrement dit, l'accent était surtout mis sur l'action. En revanche, le shéma de Bajazet se base sur le lieu qui devient l'arme tragique.

L'importance de la notion de lieu dans cette tragédie est rendue possible grâce à sa relation constante avec la notion d'espace. Cette relation est illustrée par un conflit intérieur/extérieur dont le coeur est le sérail. Il s'agit du lieu fermé par excellence qui étouffe progressivement ses victimes. D'autre part, on remarque autour du sérail la circularité de l'action qui est causée par plusieurs lieux ayant tous une fonction symbolique.

Le but de cette étude sera de démontrer comment Racine a utilisé les lieux et l'espace afin de mettre en scène l'etouffement progressif ainsi que l'insoutenable atmosphère de cette "chronique de morts annoncées"3 que ne désap- 
prouverait pas Gabriel García Marquez.

Lorsque Racine choisit de situer sa nouvelle tragédie en Turquie, il ne veut pas simplement suivre la mode orientale qui dominait à cette époque. En effet, et comme l'a écrit Philippe Hourcade, on aurait tort de croire que le poète avait pour but de créer sa propre version du Bourgeois Gentilhomme (75). Ce qui intéresse Racine est de trouver un endroit différent où il pourra développer le thème de l'étouffement hermétique autour duquel il a décidé d'organiser son intrigue. La Turquie s'impose logiquement comme le meilleur choix. Il s'agit d'un lieu qui se distingue de l'Antiquité et qui, malgré l'indéniable curiosité qu'il suscite, reste méconnu et entretient le mystérieux. Enfin, grâce à ce sérail oriental, Racine maintient la distance entre le public et sa pièce. Son objectif est de renforcer la séparation intérieur/extérieur. Il veut mettre en valeur la circularité de l'étouffement qui se resserre progressivement autour des personnages du sérail. D'ailleurs, Racine a pris soin de justifier ses choix dans les deux préfaces de la pièce. Selon lui, la distance peut se substituer au temps. Ainsi, il écrit dans la deuxième préface que "l'éloignement des pays sépare en quelque sorte la trop grande proximité des temps[...]" (Racine 10).

Cependant, Racine aborde aussi l'image de ces gens livrés à eux-mêmes dans l'enceinte fermée: "Nous avons si peu de commerce avec les princes et les autres personnages qui vivent dans le sérail que nous les considérons, pour ainsi dire, comme des gens qui vivent dans un autre siècle que le nôtre." Pour Racine, la notion d'espace remplace celle de temps. En effet, le dramaturge insiste sur la séparation des deux mondes, sur le fait que, dans le sérail, des hommes et des femmes devront prendre une décision sous le joug insupportable que représente le regard des autres.

Avant d'arriver à l'analyse du sérail et du schéma étouffant qu'il suscite, il est nécessaire de se pencher sur l'organisation technique de l'oeuvre. Comment Racine a-t-il mis en scène Bajazet? Comment a-t-il exploité la scène et les décors? Quels sont les mots ou expressions qui renforcent l'importance de la notion de lieu? Tout d'abord, qu'ils s'appellent Barthes, Maskell ou Scherer ${ }^{4}$ les critiques soulignent judicieusement l'organisation de l'espace scénique. On relève donc dans Bajazet une double relation entre "scenic place' [which] is the place represented by the stage and its immediate environs [and] 'geographical place' [which] is the locality where the action is said to unfold" (Maskell 18). Dans Bajazet, l'espace scénique a évidemment pour cadre le sérail. Les autres salles du palais ainsi que le port et le camp d'Amurat représentent l'espace géographique. Il s'agit en fait de l'espace extérieur que Roland Barthes a partagé en trois: la mort, la fuite, l'événement (5). Ainsi, Bajazet, Roxane et Atalide meurent tous les trois dans des salles ou couloirs annexes au sérail. Acomat obtient sa liberté en fuyant le sérail sur un bateau. Enfin, Amurat organise toute sa stratégie qui provoquera la tuerie finale depuis un camp matériellement invisible.

En ce qui concerne le décor, Laurent le décrit comme "un salon à la Turque."5 On imagine l'abondance luxuriante et colorée d'étoffes qui remplissent la scène. Cela ajoute au ton d'exotisme et d'étrange que Racine semble 
vouloir donner à son oeuvre. Enfin, Alain Niderst note en parlant du palais qu'"une éternelle nuit semble y régner" (89). Selon Niderst, et il n'est pas le seul, 6 l'atmosphère de Bajazet réclame un conflit entre l'obscurité du palais et du sérail en particulier et la lumière venue de l'extérieur. Dès le premier acte, Acomat déclare:

Souffrez que Bajazet voie enfin la lumière:

Des murs de ce palais ouvrez-lui la barrière; (237-38)

En réalité, si Racine ne donne pas beaucoup d'indications scéniques, une analyse lexicale de Bajazet s'avère très utile pour reconstituer la scène et le décor de la pièce. De nombreux critiques ont suivi le texte de près, mais ils n'ont pas cependant décomposé la pièce de Racine du point de vue lexical. Pourtant, il semble qu'une étude approfondie du vocabulaire de Bajazet soit une formidable source d'informations concernant non seulement la représentation des lieux mais aussi de l'atmosphère scénique.

La concordance de Freeman ${ }^{7}$ confirme l'importance des lieux dans Bajazet. Ainsi, le mot "lieu(x)" apparaît seize fois dans la pièce. De plus, une analyse stricte du vocabulaire ayant un rapport avec le lieu ou l'espace permet de distinguer plusieurs groupes lexicaux qui définissent le schéma de Bajazet. Premièrement, se retrouvent les bâtisses qui sont les témoins de l'action: "palais" (15) (ce mot ne revient autant que dans Britannicus) et "sérail" (12). Ensuite, la liaison à l'intérieur du palais et du sérail s'effectue grâce à des "chemins" (7), des portes (6), ou des détours (3). S'adressant à Osmin, Acomat dit:

Nourri dans le sérail, j'en connais les détours; (1424)

Néanmoins, les déplacements doivent souvent faire face à des obstacles (6), des murs (5), et des remparts (2). A cet égard, la phrase d'Acomat est frappante:

Des murs de ce palais ouvrez-lui la barrière; (238)

Par conséquent, si l'évolution est possible à travers les salles du palais, elle demeure limitée à ce lieu. Pour les personnages de Bajazet, le sérail est une prison dans laquelle ils restent libres de leurs mouvements et de leurs décisions. En effet, la rapidité et la fréquence des mouvements au sein du sérail se traduisent par la répétition de "cours" et de ses dérivés (5) tout comme par deux oppositions lexicales: "ouvrir" (5) et "fermer" (7) ainsi que "sortir" (10) et "entrer" (7). Donc, les personnages de Bajazet courent dans tous les sens afin d'échapper à leurs destins à l'image d'Acomat qui s'exclame:

Montrez-moi le chemin, j'y cours. (1359)

Selon le texte de Racine, le conflit majeur que doivent combattre les protagonistes de la pièce ne fait aucun doute: il s'agit de l'opposition entre la vie et la mort. Jamais ces deux mots n'ont été autant cités dans toute l'oeuvre de Racine (33 et 36 fois). Malgré la balance entre ces deux chiffres, la mort finit par l'emporter dans le palais comme si "fermer" signifiait la mise à mort. Dans l'acte II, Roxane rappelle à Bajazet l'emprise qu'elle exerce sur sa vie:

Que je puis vous l'ouvrir ou fermer pour jamais;

Que j'ai sur votre vie un empire suprême;

Que vous ne respirez qu'autant que je vous aime ? (508-10) 
Cependant, le mot qui revient le plus souvent dans Bajazet est "yeux" (38). Associé à "silence" (10), il pèse de façon insoutenable sur le sérail. Il sera expliqué plus tard comment la question du choix effectué sous le regard d'autrui peut représenter l'élément capital de Bajazet. 8

Auparavant, il est nécessaire de marquer la séparation entre le monde intérieur et le monde extérieur dans cette oeuvre. En fait, Barthes définit cette opposition comme une ambiguïte "organique" servant de base à la pièce (97). Il est alors facile de constater que l'importance et l'ampleur prises par le sérail (ou monde intérieur) dépendent en grande partie du monde extérieur représenté par la mer, le peuple, le camp d'Amurat et les muets. Plusieurs éléments renforcent cette opposition directe. En premier lieu, la mer vient toucher le palais immédiatement, sans qu'il y ait un espace entre les deux comme le soulignent respectivement Zatime et Acomat:

Et, quoique sur la mer la porte fût fermée, (1098)

Et jusqu'au pied des murs que la mer vient laver, (1719)

Ainsi, il est facile de voir le sérail comme un lieu fermé et sombre, synonyme de mort. D'ailleurs, selon Acomat:

Ce palais est tout plein...

Oui, d'esclaves obscurs,

Nourris loin de la guerre, à l'ombre de ses murs; (1415-16)

Il s'agit donc d'un site assailli par le monde extérieur, un monde de lumière que traduit cette phrase de Zatime:

Orcan, le plus fidèle à servir ses desseins,

Né sous le ciel brûlant des plus noirs Africains. (1103-04)

D'autre part, l'agitation, la multiplicité des mouvements du sérail contrastent avec le silence de tout ce qui arrive de l'extérieur. La mer ne semble pas particulièrement agitée, et Amurat reste muet à l'exemple de ses messagers. Pourtant, malgré son silence et son absence de la scène, le monde extérieur garde le contrôle tout au long de la pièce. Même Roxane se laisse aveugler par son tempérament fougueux:

Songez-vous que je tiens les portes du palais? (507)

Mais c'est bien Amurat qui gouverne depuis son camp éloigné. Il n'a aucun mal à infiltrer Orcan, son homme de confiance, qui agira comme un détonateur à l'intérieur du sérail. Le problème du sérail est qu'en dépit de son énergie, il est condamné à s'auto-détruire car quoiqu'il tente, il sera toujours privé de ce qui renforce le pouvoir extérieur: l'ouverture spatiale. ${ }^{9}$

Néanmoins, afin de rendre efficace l'opposition intérieur et extérieur dans Bajazet, Racine a créé un lien entre ces deux mondes par l'intermédiaire d'Acomat. En effet, il est le seul personnage de la pièce à braver les deux univers. Même Orcan, qui pourtant vient de l'extérieur, ne survivra pas au sérail. Osmin résume sa chute:

Transportés à la fois de douleur et de rage,

Nos bras impatients ont puni son forfait,

Et vengé dans son sang, la mort de Bajazet. (1690-92)

En fait, comme l'écrit Roland Barthes, sortir du sérail signifie la mort pour tous 
sauf pour Acomat (103). Il faut donc admettre la dualité de ces deux mondes, offrant l'image d'une arme à double tranchant. Evert Van Der Starre résume bien cette théorie lorsqu'il écrit que "les murs [du sérail] protègent les conspirateurs contre le danger qui vient de la mer; mais ils sont aussi une entrave à la liberté dont la mer est une promesse; qui ne se réalisera que pour Acomat" (89).

Dans sa pièce, le dramaturge met en scène un itinéraire progressif qui mêle les lieux aux sentiments. Tout au long de l'oeuvre, les personnages sont les victimes d'un "étau" mortel et étouffant qui se resserre inexorablement. Racine utilise un axe circulaire dont le centre est le sérail. Les protagonistes y "tournent en rond" tout en s'épiant les uns les autres. Le climax de l'oeuvre se traduit par une tension optimale qui provoquera la tuerie finale. L'idée de cet itinéraire tragique à travers les couloirs du sérail et du palais ne fait aucun doute dès le début de la pièce. Comme mentionné auparavant, Bajazet est une "chronique de morts annoncées." Racine a donc inclu la prédestination. ${ }^{10}$ Dans l'acte II, Roxane dit à Bajazet:

De toi dépend ma joie et ma félicité:

De ma sanglante mort ta mort sera suivie. (556-57)

Cependant, Roxane ne pensait pas succomber après Bajazet. L'ordre des mots dans le vers 557 s'avère très important car il démontre que Roxane pense déjà en termes de pouvoir. Elle est sûre de dominer le palais et cette assurance aveugle l'empêche de sentir le danger qui mettra un terme à sa vie. Dans tous les cas, lorsque Roxane prononce ces vers annonciateurs, elle se trouve déjà avec Bajazet et Atalide dans ce sérail qu'elle n'a jamais quitté. Par conséquent, c'est dans ce lieu fermé que va se dérouler l'action. Roxane donne le coup d'envoi dans l'acte II:

Sortez. Que le sérail soit désormais fermé;

Et que tout rentre ici dans l'ordre accoutumé. (571-72)

En fait, la force de suffocation ressentie dans Bajazet vient d'abord des lieux. L'extérieur représenté par le camp d'Amurat et la mer pèsent sur la ville de Byzance qui encercle le palais qui abrite le sérail qui détient les protagonistes. Dans Bérénice, le peuplé de Rome presse Titus pour ne pas qu'il épouse une reine. A l'intérieur de Bajazet, ce sont les lieux qui étouffent les personnages. Les critiques ${ }^{11}$ sont d'accord pour affirmer qu'en étant encerclé, le sérail devient un labyrinthe. En effet, cet endroit pullule de couloirs et de chemins dans lesquels s'épuisent les personnages. Pour Roxane, la passion se transforme en obsession destructrice. Elle est victime du sérail malgré elle, prise à son propre piège. Le sort qu'elle réservait à Bajazet lui est infligé par Amurat. Pendant que Roxane, Bajazet et Atalide s'épuisent à heurter leurs divergences sentimentales à l'intérieur du sérail, la mort venue de l'extérieur, progresse à grands pas. Orcan le muet débarque dans la ville et force un par un les obstacles pour arriver jusqu'au sérail. Le fait que ce soit le muèt Orcan qui enclenche la tuerie finale en assassinant Roxane est très important. En effet, Orcan ou son chef Amurat viennent de l'extérieur, sont silencieux et n'apparaissent jamais sur scène. Ils rappellent la froideur des murs du sérail à cause de leur silence. C'est comme si c'était le lieu qui tuait. 
Par ailleurs, l'etouffement du lieu provoque chez les personnages de Bajazet la question du choix. Le temps presse, on a de plus en plus de mal à supporter cet emprisonnement, on doit choisir. Bajazet doit épouser Roxane contre son gré ou mourir. Le dilemme de Roxane oppose la gloire et Amurat à l'amour pour Bajazet. Atalide attend et n'aura que Bajazet ou la mort. Non seulement les "prisonniers" du sérail sont victimes de l'encerclement du lieu mais ils sont surtout à la merci des autres. Constamment sous l'épreuve de l'oeil d'autrui, ils doivent penser à ce choix entre la vie et la mort. Roland Barthes a bien remarque l'importance du regard: "The seraglio is rather like an arena in which Roxanne is the matador: she must kill, but under the eyes of an invisible judge who surrounds and watches her" (97). La solitude de l'être livré à lui-même sous l'oeil glacial, quasi "emmuré" de l'autre ressort du sérail de Bajazet. Les "yeux" n'apparaissent pas moins de trente-huit fois dans la pièce (Freeman, 1418-19). Parfois, on doit subir directement l'oeil de l'autre. Tel est le cas de Roxane qui veut confronter Bajazet à son regard:

Sans être préparé se présente à mes yeux. (332)

Cependant les vers qui démontrent bien l'importance des yeux d'autrui comme obstacles inaccessibles et destructeurs sont prononcés par Osmin dans la première scène de l'acte I:

Mais pouvaient-ils tromper tant de jaloux regards

Qui semblent mettre entre eux d'invincibles remparts? (14344)

Dans le sérail, les regards ont vite fait de s'entrecroiser et rien n'y pourra changer. A l'intérieur de ce lieu auto-destructif, le choix est impossible. Les protagonistes n'ont aucun contrôle sur le sérail que dirige en fait le monde extérieur d'Amurat.

Le seul recours désespéré du trio emprisonné est de s'adresser au ciel. On se dit qu'une prière est impalpable et qu'elle peut donc traverser les murs opaques du sérail. Néanmoins, aucun ange salvateur n'arrivera. Pourtant, on ne cesse d'invoquer le "ciel" avec pas moins de trente et une citations dans le texte (Freeman 205). En guise d'exemple, citons ces interventions d'Atalide:

L'occasion, le ciel pourra vous les dicter. (788)

Le ciel, le juste ciel vous devait ce miracle. (957)

A défaut d'autre chose, on en est réduit à appeler le ciel en renfort mais le Tout Puissant ne répond pas et on doit bien se rendre à l'évidence. Avec le ciel, Racine nous propose l'opposition finale, la plus pesante, la plus ambitieuse: le sérail cloisonné face, non pas au Tout Puissant, mais au ciel, l'immensité spatiale. C'est la petitesse de l'Homme livré à lui-même dans le temps présent, encombré de questions sans réponses, opposé à l'espace universel. En effet, il n'y a point de miracle pour éviter la tuerie finale de Bajazet. Au contraire, on y remarque l'impuissance de l'Homme dans un espace minimisé sur lequel il n'a aucun contrôle. Quoiqu'il fasse, quoiqu'il dise, il est à la merci de son environnement; il ne peut pas changer sa destinée.

Le sérail de Bajazet met en valeur une circularité étouffante qui emprisonne le trio Roxane-Bajazet-Atalide dès le début de la pièce. De la même façon 
que l'espace extérieur encercle le sérail, le regard de chaque personnage pèse sur autrui à l'image d'un mur. La question du choix est bien réelle mais elle demeure irrésolue car sans réponse absolue. En effet, Roxane et Bajazet mourront quoiqu'ils choisissent, l'amour, le pouvoir ou l'honneur. L'Homme se trouve dans une situation identique car il peut opter pour le camp de Dieu ou pour celui des athés mais il reste impuissant face à sa destinée. Le sérail est donc le Monde tel que l'Homme l'a toujours connu: unique et inexpliqué.

University of North Carolina at Chapel Hill 


\section{Notes}

1 Cf. Sophie Wilma Deierkauf-Holsboer, Le Théatre de l'Hôtel de Bourgogne, vol. 2 (Paris: Nizet, 1970), 144-45.

2 Cf. première préface de Racine dans l'édition de Xavier de Courville, (Paris: Seuil, 1947), 7.

3 Citation empruntée au titre de l'oeuvre de Gabriel García Marquez.

4 Cf. respectivement Sur Racine (Paris: Seuil, 1963); Racine. A Theatrical Reading (Oxford: Clarendon, 1991); Racine: 'Bajazet' (Paris: CDU, 1958).

5 Cf. David Maskell, "La Précision du Lieu" dans: Christine M. Hill, Racine: Théâtre et Poésie, (Leeds: Cairns, 1991), 159.

6 Cf. Noémi Hepp, "Autour de 'Bajazet" dans: L'Information Littéraire 38-2 (1986): 62.

${ }^{7}$ Toutes les références concernant la fréquence des citations sont issues de la Concordance de Bryant Freeman. Elles sont mentionnées entre parenthèses à la suite de chaque mot concerné. Afin de donner plus de clarté au texte, les numéros de page de la Concordance ne sont pas idiquées. Bryant Freeman a classé les expressions par ordre alphabétique dans ses deux volumes.

8 Pour une plus ample étude lexicale de Bajazet, on pourra lire l'excellent livre de Jacques-Gabriel Cahen, Le Vocabulaire de Racine (Genève: Slatkine, 1970), 121-127.

${ }^{9}$ Cf. Philippe Butler, Classicisme et Baroque dans l'Oeuvre de Racine (Paris: n.p.,1959), 132-33.

10 Raymond Picard développe cette même thèse de la condamnation des personnages dès le début de la pièce. Oeuvres Complètes, Jean Racine, La Pléiade (Paris: Gallimard, 1950) préface de Bajazet.

${ }^{11}$ Starre et Cahen en particulier. 


\section{Ouvrages Cités}

Barthes, Roland. On Racine. Trans. Richard Howard. New York: Octagon, 1977.

Butler, Philippe. Classicisme et Baroque dans l'Oeuvre de Racine. Paris: n.p., 1959.

Cahen, Jacques-Gabriel. Le Vocabulaire de Racine. Genève: Slatkine, 1970. Deierkauf-Holsboer, Sophie Wilma. Le Théâtre de l'Hôtel de Bourgogne. 2 vols. Paris: Nizet, 1970.

Freeman, Bryant and Alan Batson. Concordance du Théâtre et des Poésies de Jean Racine. 2 vols. Ithaca: Cornell UP, 1968.

Hepp, Noémi. "Autour de Bajazet." L'Information Littéraire 38.2 (1986): 6166.

Hourcade, Philippe. "Racine et le Goût du Spectacle dans Bérénice et Bajazet." Jeunesse de Racine (1968): 66-82.

Lapp, John C. Aspects of Racinian Tragedy. Toronto: U of Toronto P, 1955.

---. "La Précision du Lieu." Racine: Théâtre et Poésie: Actes du Troisième Colloque Vinaver, Manchester 1987. Ed. Christine M. Hill. Leeds: Cairns, 1991. 151-171.

Niderst, Alain. Les Tragédies de Racine. Diversité et Unité. Paris: Nizet, 1975.

Picard, Raymond, ed. Oeuvres Complètes. By Jean Racine. Vol. 1 Bibliothèque de la Pléiade. Paris: Gallimard, 1950.

Racine, Jean. Bajazet. Ed. Xavier de Courville. Collection "Mises en scène." Paris: Seuil, 1947.

Schérer, Jacques. Racine: Bajazet. Les Cours de Sorbonne; Littérature Française. Paris: Centre de Documentation Universitaire, 1958.

Starre, Evert Van der. Racine et le Théâtre de l'Ambigüté, Etude sur Bajazet. Leiden: Universitaire Pers Leiden, 1966. 


\section{LES PAS}

Tes pas, enfants de mon silence,

Saintement, lentement placés,

Vers le lit de ma vigilance

Procèdent muets et glacés.

Personne pure, ombre divine,

Qu'ils sont doux, tes pas retenus!

Dieux!... tous les dons que je devine

Viennent à moi sur ces pieds nus!

$\mathrm{Si}$, de tes lèvres avancées, Tu prépares pour l' apaiser, A l'habitant de mes pensées

La nourriture d'un baiser.

Ne hâte pas cet acte tendre,

Douceur d'être et de n'être pas, Car j'ai vécu de vous attendre, Et mon coeur n'était que vos pas.

-Paul Valéry, Charmes, 1922 